\title{
DETERIORO COGNITIVO ASOCIADO A RADIOTERAPIA EN TUMORES CEREBRALES
}

\section{COGNITIVE IMPAIRMENT ASSOCIATED WITH RADIOTHERAPY FOR BRAIN TUMORS}

\author{
Rubén Pérez-Elvira, María Clavel \\ Servicio de Neuro-rehabilitación. Hospital Centro de Cuidados Laguna
}

Resumen

Introducción: Existe un limitado conocimiento acerca de la influencia sobre las funciones cognitivas de los tratamientos para los tumores cerebrales basados en la radioterapia (RT).

Objetivo: Realizar una revisión actualizada de los trabajos desarrollados en los últimos años acerca de los efectos de la RT sobre la cognición en los tumores cerebrales.

Método: Mediante el software EndNote X5 se extrajeron los artículos que contenían "radiotherapy" y "cognition" tanto en su título, como en palabras claves, abstracts o contenidos en los años 2009-2011. Asimismo, se realizó una búsqueda ampliada a través de las referencias de los artículos extraídos. Se descartaron aquellos artículos en los que no se reflejase la relación entre $\mathrm{RT}$ y el desarrollo de alteraciones cognitivas o no se centraran en tumores cerebrales.

Resultados: 14 artículos fueron seleccionados: 7 originales, 6 revisiones y 1 artículo de opinión. Los hallazgos se centraron en 3 aspectos: a) alteraciones cognitivas debidas a la RT; b) factores de riesgo para el desarrollo de deterioro cognitivo inducido por la RT; c) tiempo transcurrido desde la aplicación de la RT hasta la aparición de las alteraciones cognitivas.

Conclusiones: Los nuevos estudios confirman hallazgos de las últimas décadas, principalmente que las funciones más comúnmen-

\section{Abstract}

Introduction: There is limited knowledge about the influence on cognitive functions of the treatments for brain tumors based on radiotherapy (RT).

Objective: To review the works conducted in recent years about the effects of RT on cognition in brain tumors.

Method: Using EndNote software X5, articles containing "radiotherapy" and "cognition" in its title, keywords, abstracts or main text in the years 2009-2011 were extracted. Also, an extensive search through the references of articles taken was done. Those works that did not reflect the relationship between RT and the development of cognitive impairment or did not focus on brain tumors were disregarded.

Results: 14 articles were selected: 7 originals, 6 reviews, and 1 opinion article. The findings focused on three aspects: a) cognitive impairment due to the RT, b) risk factors for cognitive impairment induced by RT c) time from the application of RT to the onset of cognitive impairment.

Conclusions: The new studies confirm findings of recent decades, namely that the functions most commonly affected are attention, memory and learning, psychomotor and executive functions; that there are identifiable risk factors for cognitive impairment associated to RT; and that alterations in cognitive

Correspondencia:

María Clavel Claver

Coordinadora Servicio de Neuro-rehabilitación

Hospital Centro de Cuidados Laguna

C/ Concejal Francisco José Jiménez, 128 - 28047 MADRID

Email: mclavel@lagunacuida.org 
te afectadas son la atención, la memoria y el aprendizaje, la psicomotricidad y las funciones ejecutivas; que existen algunos factores de riesgo identificables para el desarrollo de deterioro cognitivo asociado a la RT; así como que las alteraciones en la función cognitiva tras la RT suelen aparecer de manera evidente después un largo período de tiempo.

Palabras clave: Neuropsicología, radioterapia, deterioro cognitivo. function after RT usually occur after a long period of time.

Keywords: Neuropsychology, radiotherapy, cognitive impairment.

\section{PLANTEAMIENTO DEL PROBLEMA}

El número de pacientes que sobreviven al cáncer ha aumentado en las últimas décadas debido, entre otros motivos, a la precocidad del diagnóstico y a los tratamientos disponibles. En España, los tumores cerebrales suponen el $2 \%$ de todos los tumores ${ }^{(1)}$ y tienen una incidencia de 7,5 por cada 100000 habitantes. En la distribución por sexos se observa un ligero predominio de los tumores benignos en mujeres, mientras que los malignos y las cifras globales son mayores en varones ${ }^{(2)}$. La incidencia aumenta de forma notable con la edad en ambos sexos, principalmente entre los 75 y 85 años, siendo el glioblastoma multiforme y el astrocitoma ${ }^{(2)}$ los tipos de tumor más frecuente en población geriátrica.

Los tratamientos empleados en esta tipología de tumores, principalmente la quimioterapia, radioterapia (RT) y la radioquimioterapia, han demostrado su eficacia con mayores índices de supervivencia, pero son frecuentes y conocidos los efectos secundarios que provocan, entre otros, en el sistema nervioso central como alteraciones en las funciones cognitivas superiores en los pacientes que los reciben ${ }^{(3-7)} \mathrm{e}$ influyen, por tanto, en el correcto desempeño de su vida diaria y en la calidad de la misma ${ }^{(6,8,9)}$. Por todo esto, cada vez toma más importancia dilucidar los efectos que estas terapias tienen sobre la cognición. La influencia sobre las funciones cognitivas de los tratamientos para los tumores cerebrales basados en la quimioterapia han sido más estudiadas que los efectos de la RT, que no han sido objeto de investigación hasta hace relativamente poco tiempo y, por tanto, existen pocos artículos científicos disponibles acerca del tema.

La RT tiene un importante papel en el tratamiento de pacientes con tumores cerebrales primarios o por metástasis ${ }^{(3)}$, tanto en población adulta como pediátrica. Pero, a pesar de su eficacia como tratamiento anticanceroso, la radiación suele dañar también tejidos sanos afectando, como se ha mencionado, a la cognición. De hecho, la radiación cerebral global tiene un amplio efecto negativo sobre la cognición, por lo que en las últimas décadas el tratamiento de RT focal ha sido de primera de elección para ciertos tipos de tumores ${ }^{(3)}$.

Existe una manifiesta escasez de estudios clínicos que relacionen la RT y la función cognitiva en los tumores cerebrales ${ }^{(10)}$. Nuestro propósito en este artículo es realizar una actualización en castellano de los trabajos desarrollados en los últimos años acerca de los efectos de la RT sobre la cognición en los tumores cerebrales.

La revisión se ha realizado mediante el software EndNote X5, un software para la búsqueda automática de documentos en bases de datos. Como criterios de búsqueda se introdujeron los siguientes parámetros: 
1.- En el campo "años" se introdujo el intervalo entre "2009-2011", ajustando así la búsqueda a los dos últimos años, criterio comúnmente utilizado en las revisiones bibliográficas.

2.- Los términos radiotherapy y cognition fueron introducidos para que el software arrojase todos los artículos que contuviesen dichos términos, tanto en su título, como en sus palabras claves, abstracts o contenidos.

3.- Asimismo, e impuesto por la propia dinámica de revisión de dichos artículos, se realizó una búsqueda complementaria de otros anteriores considerados impres- cindibles por los autores, que les citaban insistentemente, o que ahora se desdecían de sus conclusiones.

4.- Se eliminaron aquellos artículos en cuyo abstract no se reflejase claramente la relación entre RT y el desarrollo de alteraciones cognitivas, así como aquellos que no se centraron en tumores cerebrales.

Tras eliminar los artículos que no cumplieron los requisitos de la búsqueda, el número total de trabajos fue de 14, agrupados en tres categorías: originales, revisiones y otros, según se muestran en la Tabla 1. En la Tabla 2 se pueden observar las características de los originales.

\section{Tabla 1. Trabajos (originales y de revisión) sobre los efectos de la radioterapia en las funciones cognitivas en pacientes con cáncer cerebral}

\begin{tabular}{|c|c|c|}
\hline Autores y Año & Tipo de Artículo & Revista \\
\hline Lawrence et al. $2010^{(3)}$ & $\begin{array}{l}\text { Revisión } \\
\text { Suplemento }\end{array}$ & $\begin{array}{c}\text { International Journal of Radiation } \\
\text { Oncology/Biology/Physics }\end{array}$ \\
\hline Douw et al. $2009^{(4)}$ & Original & The Lancet Neurology \\
\hline Welzel et al. 2008(5) & Original & Strahlentherapie und Onkologie \\
\hline Rigal et al. $2011^{(6)}$ & Revisión & Psycho-Oncology \\
\hline Brown y Cerhan 2009 $9^{(13)}$ & $\begin{array}{l}\text { Otros: Reflection } \\
\text { and Reaction }\end{array}$ & The Lancet Neurology \\
\hline Jalali et al., 2010 & Original & $\begin{array}{c}\text { International Journal of Radiation } \\
\text { Oncology/Biology/Physics }\end{array}$ \\
\hline Platta et al. $2010^{(12)}$ & Revisión & $\begin{array}{c}\text { American Journal of Clinical On- } \\
\text { cology }\end{array}$ \\
\hline Merchant et al. 2009(7) & Original & Journal of Clinical Oncology \\
\hline Shibamoto et al. $2009^{(11)}$ & Revisión & $\begin{array}{l}\text { International journal of Clinical } \\
\text { Oncology }\end{array}$ \\
\hline Duffner, $2010^{(3)}$ & Revisión & $\begin{array}{c}\text { European Journal of Paediatric } \\
\text { Neurology }\end{array}$ \\
\hline Corn et al. 2009(3) & Original & Journal of Neurooncology \\
\hline Hilverda et al. $2010^{(15)}$ & Original & Journal of Neurooncology \\
\hline Chang et al. 2009 & Original & The Lancet Oncology \\
\hline Van den Brent et al. 2011 ${ }^{(11)}$ & Revisión & The Lancet Oncology \\
\hline
\end{tabular}




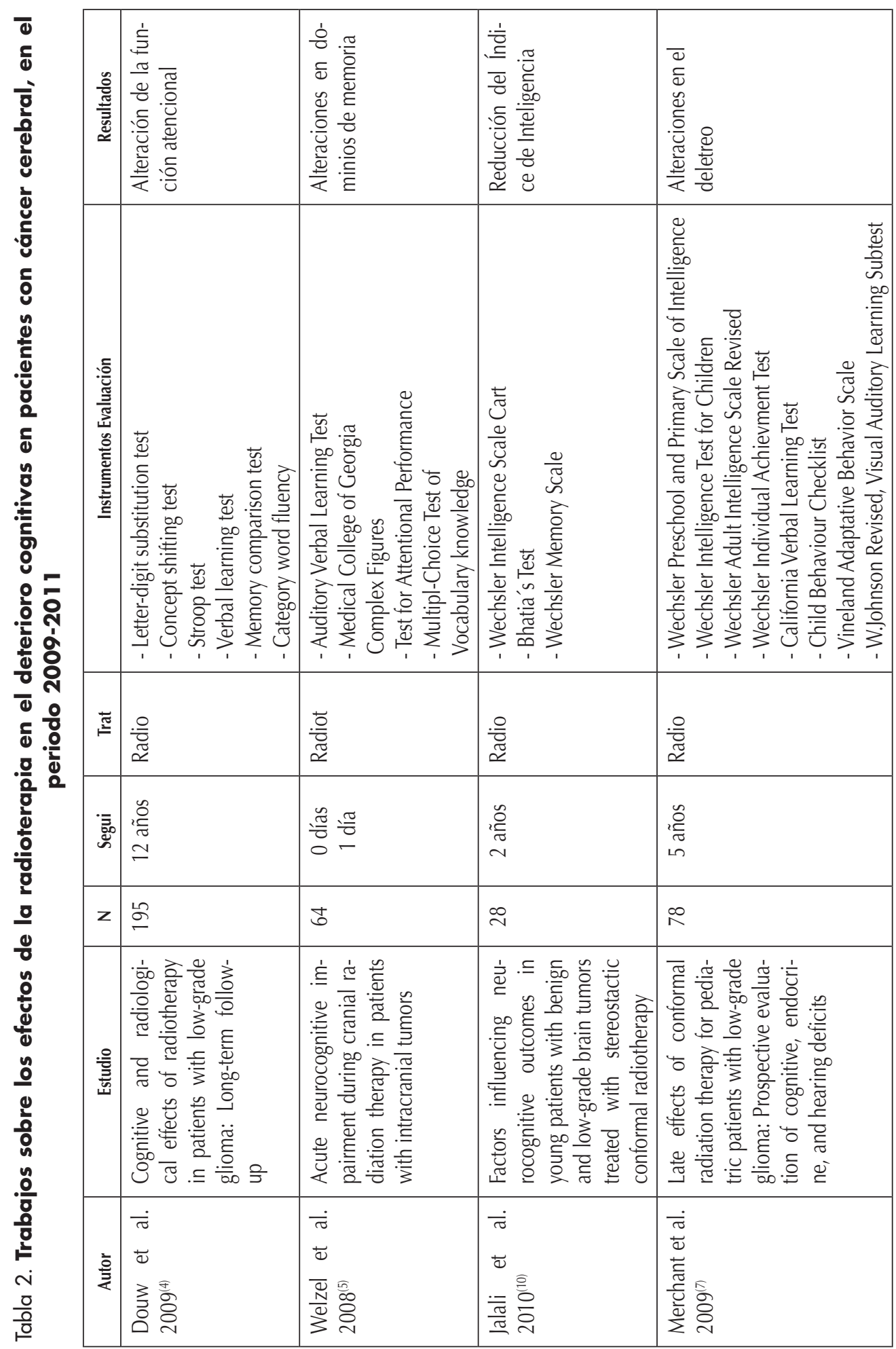




\begin{tabular}{|c|c|c|c|}
\hline 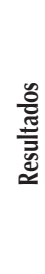 & 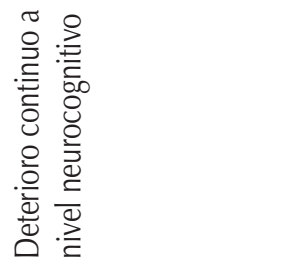 & 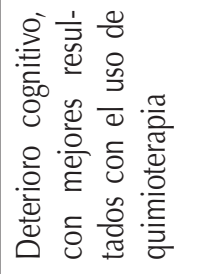 & 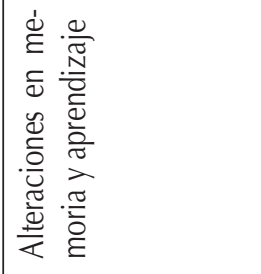 \\
\hline 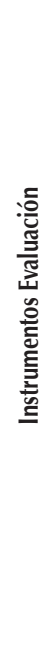 & 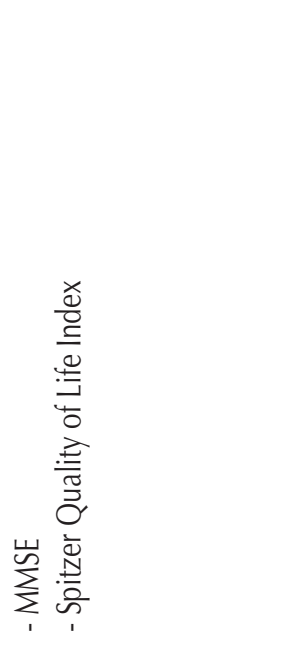 & , & 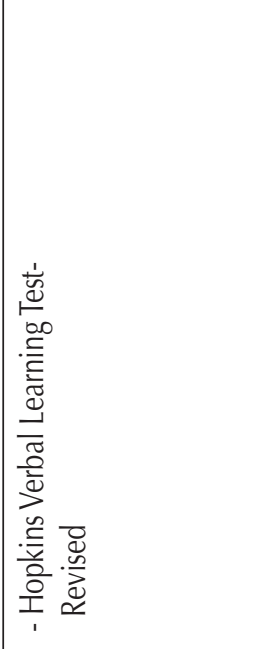 \\
\hline ঙ্ّ & 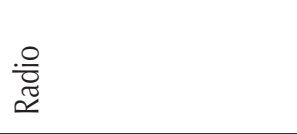 & 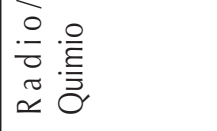 & 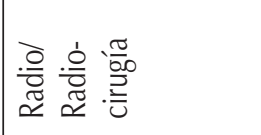 \\
\hline 信 & 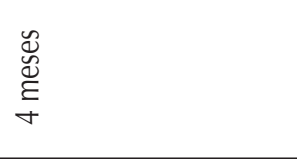 & 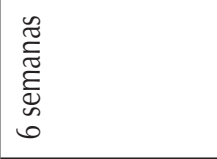 & 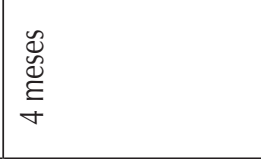 \\
\hline$z$ & i & $\stackrel{m}{=}$ & $\stackrel{\circ}{\circ}$ \\
\hline 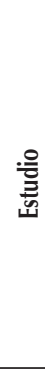 & 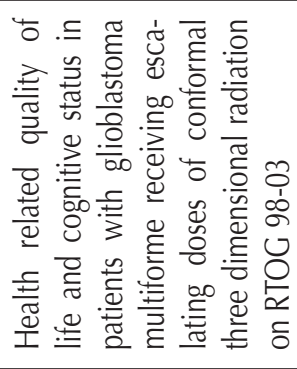 & 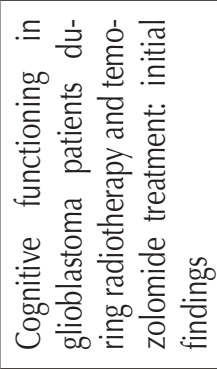 & 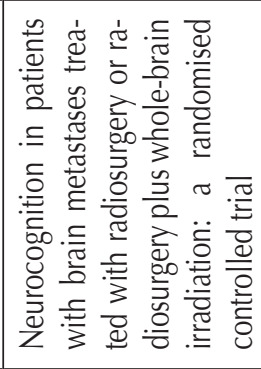 \\
\hline 言 & 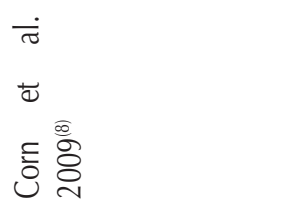 & 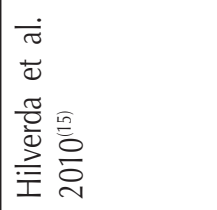 & 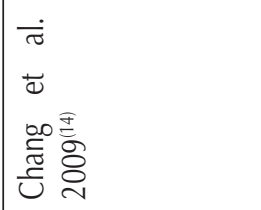 \\
\hline
\end{tabular}




\section{DESARROLLO DEL TEMA}

Los tumores cerebrales primarios tienen una incidencia del $20 \%$ en pacientes menores de 18 años $^{(3)}$ y aproximadamente el $30 \%$ de las metástasis en tumores sóli$\operatorname{dos}^{(3,11)}$.

La RT continúa siendo en la actualidad uno de los tratamientos más utilizados en pacientes con metástasis cerebral ${ }^{(11)}$. Los efectos agudos y tardíos de la RT en el cerebro son comunes y representan una fuente importante de morbilidad ${ }^{(3)}$. Independientemente de la dosis empleada, los diferentes estudios relacionados encuentran evidencia de afectación cognitiva que la RT causa en los pacientes ${ }^{(4)}$ en los que se emplea. Estos efectos negativos sobre la cognición parecen localizar su origen en lesiones vasculares por daños en la sustancia blanca, desmielinización y necrosis ${ }^{(12)}$.

Tres son los núcleos temáticos que jalonan la esfera cognitiva en la literatura revisada: a) alteraciones cognitivas debidas a la RT, b) factores de riesgo para el desarrollo de deterioro cognitivo inducido por la RT y c) tiempo transcurrido desde la aplicación de la RT hasta la aparición de las alteraciones cognitivas.

\section{a) Alteraciones Cognitivas debidas a la RT}

La mayoría de los trabajos sobre los efectos negativos de la RT y la quimioterapia en el funcionamiento cognitivo de las últimas décadas informaban sobre alteraciones en la atención, la memoria, las funciones ejecutivas y las funciones premotoras. Este patrón sigue manteniéndose en los estudios más recientes.

- Según los resultados del estudio de Douw et al. ${ }^{(4)}$, los pacientes con tumores cerebrales tratados con RT, frente a aqueIlos que no recibieron este tratamiento, mostraron alteraciones de la función atencional, pero no se observaron diferencias significativas en dominios como la veloci- dad de procesamiento de la información, funcionamiento psicomotor o memoria de trabajo. Estos autores ${ }^{(4)}$ teorizan que dosis mayores de radiación podrían acelerar la ocurrencia de los déficits. Brown y Cerhan $^{(13)}$ afirman que los datos ofrecidos por Douw et al.(4) no son generalizables a los tratamientos de RT actuales, ya que su muestra se compone de pacientes que recibieron RT en los años setenta, y las técnicas de RT utilizadas en esa época, ya obsoletas, empleaban dosis de radiación mayores que las actuales.

- Poco desarrollado es el estudio de las alteraciones en fases agudas. Welzel et al. ${ }^{(5)}$ informan de alteraciones a corto plazo tras la aplicación de la RT, principalmente de la memoria verbal, pero rara vez estas alteraciones se han demostrado importantes.

- Jalali et al. ${ }^{(10)}$ observaron una reducción del índice de inteligencia en población pediátrica dos años después de recibir el tratamiento de RT.

- Joly et al. ${ }^{(6)}$ sugieren que aunque las alteraciones cognitivas pueden ser sutiles, éstas tendrían repercusión sobre la memoria, la concentración, las funciones ejecutivas y la velocidad de procesamiento de la información. Estos y otros autores ${ }^{(6,9)}$ manifiestan que ni las pruebas de cribado ni los autoinformes de los pacientes, que además pueden estar contaminados por síntomas afectivos o por la fatiga, serían suficientemente sensibles para detectar tales alteraciones y que es necesaria la creación de baterías de pruebas sensibles, breves y completas para la adecuada exploración neuropsicológica ${ }^{(7)}$.

- Shibamoto et al. ${ }^{(1)}$ comunican que las alteraciones cognitivas estarían restringidas únicamente a la memoria verbal, y que la función atencional y la memoria visual no se verían afectadas. En esa misma línea, Chang et al. ${ }^{(14)}$ encontraron que, frente a los pacientes no radiados, los pacientes que habían recibido $\mathrm{RT}$ mostraban 
un desempeño peor en los dominios de memoria y aprendizaje.

- Algunos autores, como Hilverda et al. ${ }^{(15)}$, han propuesto que el tratamiento combinado de RT con temozolomida prevendría la aparición de déficits cognitivos, pero el seguimiento de los pacientes no fue lo suficientemente prolongado como para evidenciar los déficits.

En la práctica totalidad de los trabajos, los autores advierten que no se pueden excluir la influencia de otras variables sobre la cognición.

\section{b) Factores de riesgo de desarrollo de déficits cognitivos inducidos por RT}

En los pacientes pediátricos los factores de riesgo más importantes para el desarrollo de deterioro cognitivo inducido por RT, como se venía manifestando en investigaciones anteriores, son la edad, con un mayor riesgo a menor edad $(3,7,10,16)$, y el volumen de la dosis de radiación ${ }^{(4,10,11,16)}$, con mayor riego a mayor dosis. Merchant et al. ${ }^{(7)}$ afirman que el efecto de la edad excede al efecto de la dosis de radiación. Otros factores de riesgo incluyen el género femenino, extensión de la reserción quirúrgica, hidrocefalia, quimioterapia concomitante, localización del tumor y volumen de cerebro irradiado ${ }^{(3,16)}$.

La relación entre la edad y las secuelas cognitivas con radiación global del cerebro es conocida desde hace algún tiempo; sin embargo el efecto de la radiación parcial no está aún bien documentado ${ }^{(10)}$.

En los adultos, los factores de riesgo podrían incluir el volumen irradiado y un amplio intervalo temporal tras el tratamiento $^{(3,4,11)}$. Los adultos mayores de 60 años parecen ser especialmente vulnerables al declive cognitivo asociado a la RT; esta vulnerabilidad podría explicarse por cuestiones como la alta difusión del tumor, que tenga un patrón angiocéntrico de crecimiento y que la mayoría recibe altas dosis de metotrexato, que es altamente neurotóxico; por esto Lawrence et al. ${ }^{(3)}$ sugieren que debería evitarse dosis altas de radiación en personas mayores.

\section{c) Tiempo medio de aparición de los déficit cognitivos asociados a RT}

La déficits cognitivos asociados a la RT suelen aparecer a largo plazo ${ }^{(3,4,5)}$ y aunque algunos trabajos informan de la posibilidad de que estos déficits se muestren en etapas tempranas tras la aplicación de la $\mathrm{RT}$, rara vez, empero, serían graves en dichas etapas ${ }^{(5)}$.

Platta et al. ${ }^{(12)}$, afirman que los efectos negativos sobre la cognición aparecen a partir de los seis meses. Sin embargo, Lawrence et al. ${ }^{(3)}$ advierten que pocos estudios han seguido a los pacientes el tiempo suficiente para detectar las alteraciones cognitivas. Manifiestan estos autores ${ }^{(3)}$ que en las investigaciones con menos de dos años de seguimiento no se observan diferencias en cuanto al estado cognitivo entre pacientes y controles, y que en estudios con seguimientos de cinco años se pueden observar los efectos negativos de la RT en aquellos pacientes que la recibieron. Douw et al. ${ }^{(4)}$ sitúan la aparición de los déficits entre los seis y los doce años tras la aplicación de la RT.

\section{DISCUSIÓN}

La existencia de déficits cognitivos asociados a los tratamientos contra el cáncer es un hecho ampliamente probado. En concreto, los efectos negativos de la RT sobre la cognición han sido documentados tanto en el tratamiento de pacientes con tumores cerebrales, como en pacientes con tumores en regiones corporales próximas al encéfalo, como cabeza y cuello, donde el tratamiento con RT afecta también, aunque de manera parcial, al cerebro. 
Sin embargo, a pesar de ser un hecho bien documentado, la existencia de déficits cognitivos inducidos por la RT no ha sido muy estudiada en los casos de tumores cerebrales. Existe una dificultad intrínseca para el estudio de estos casos, y es que el propio tumor puede provocar sintomatología cognitiva, dificultando con ello el esclarecimiento de la naturaleza de los déficits, ¿RT o cáncer?

La falta de guías de intervención y de baterías específicas para la valoración de las funciones cognitivas en pacientes con tumores cerebrales radiados discrepa frente a la preocupación general que se recoge en la mayoría de los trabajos acerca de la necesidad de identificar e intervenir sobre los déficits cognitivos para garantizar una mejora de la calidad de vida de los pacientes.

\section{CONCLUSIÓN}

El volumen de trabajos publicados sobre el tema en los últimos dos años es manifiestamente escaso. En líneas generales, los nuevos estudios centrados en los efectos sobre la cognición de la RT en tumores cerebrales vienen a confirmar algunos de los hallazgos de las últimas décadas, principalmente que las alteraciones en la función cognitiva tras la RT suelen aparecer de manera evidente después un largo periodo de tiempo, que las funciones más comúnmente afectadas son la atención, la memoria y el aprendizaje, la psicomotricidad y las funciones ejecutivas, así como que existen algunos factores de riesgo identificables para el desarrollo de deterioro cognitivo asociado a la RT.

En la actualidad se están estudiando los efectos sobre la cognición de terapias combinadas como la radioquimioterapia o la radiocirugía combinada con RT en los tumores cerebrales. Aunque algunos autores, como Brown y Cehran ${ }^{(13)}$ afirman que los nuevos tratamientos de RT no son tan agresivos como los de hace unas décadas, la realidad evidencia que la aplicación de RT siempre aumenta el riesgo de provocar deterioro cognitivo en los pacientes.

Las nuevas líneas de investigación tienen su objetivo en caracterizar detalladamente los patrones de afectación cognitiva inducida por RT y detectar factores de riesgo más determinantes para poder así realizar una óptima selección de pacientes candidatos, o no, a la RT, así como desarrollar diseños de tratamientos con RT que minimicen los efectos cognitivos. Asimismo, también se están desarrollando trabajos centrados en los efectos profilácticos de ciertos fármacos sobre la influencia negativa de la RT en la cognición.

\section{REFERENCIAS BIBLIOGRÁFICAS}

1. Asate. Tomores cerebrales. [En línea] 2011 [Acesso 16 de octubre de 2011]. Disponible en: http://www.asate.es/tag/tumorescerebrales/

2. AECC. Tumores del sistema nervioso central [En línea] 2011 [Acces 10 de octubre de 2011] disponible en https://www.aecc. es/SobreElCancer/ CancerPorLocalizacion/ Sistemanerviosocentral/Paginas/Incidencia. aspx

3. Lawrence $Y R$, Allen Li X, El Naqa I, Hahn CA, Marks LB, Merchant TE, Dickers AP. Radiation dose-volume effects in the brain. Int. J. Radiation Oncology Bio. Phys 2010: 76: S20-S27. Doi:10.1016/j.ijrobp.2009.02.091

4. Douw L, Klein M, Fagel S, Van den Heurel J, Taphoorn M, Aaronson NK, et al. Cognitive and radiological effects of radiotherapy in patients with low-grade glioma: Long-term follow-up. Lancet Neurol 2009; 8: 810-8. Doi:10.1016/S1474-4422(09)70204-2

5. Welzel G, Fleckenstein K, Mai SK, Hermann B, Kraus-Tiefenbacher U, Wenz F. Acute neurocognitive impairment during cranial radiation therapy in patients with 
intracranial tumors. Strahlenther Onkol., 200884 (12):647-54. Doi: 10.1007/ s00066-008-1830-6

6. Rigal JF, Noal S, Giffard B. Cognitive dysfunction and cancer: Which consequences in terms of disease managment?. Psychooncology 2011, En prensa. Doi-10.1002/ pon.1903.

7. Merchant TE, Conklin HM, Wu J, Lustig $\mathrm{RH}$, Xiong X. Late effects of conformal radiation therapy for pediatric patients with low-grade glioma: Prospective evaluation of cognitive, endocrine, and hearing deficits. J Clin Oncol 2009; 27: 3691-7. Doi:10.1200/JCO.2008.21.2738

8. Corn, BW, Wang, M, Fox, S, Michalski, J, Purdy, J, Simpson, J, et al. Health related quality of life and cognitive status in patients with glioblastoma multiforme receiving escalating doses of conformal three dimensional radiation on RTOG 98-03. J Neurooncol 2009; 95: 247-57. Doi:10.1007/s11060-009-9923-3

9. Van den Brent MJ, Wefel JS, Schiff D, Taphoorn MJB, Jaeckle K, Junck L, et al. Response assessment in neuro-oncology (a report of the RANO group): assessment of outcome in trials of diffuse low-grade gliomas Lancet Oncol 2011; 12: 583-93. Doi:10.1016/S1470-2045(11)70057-2

10. Jalali R, Mallick I, Dutta D, Garwami S, Gupta T, Munshi A, Deshpande D, Sarin R. Factors influencing neurocognitive outcomes in young patients with benign and low-grade brain tumors treated with stereostactic conformal radiotherapy. Int J Rad Oncol Biol Phys 2010; 77; 974-9. Doi:10.1016/j.ijrobp.2009.06.025

11. Shibamoto Y, Sugie C, Iwata, H. Radiotherapy for metastatic brain tumors. Int J Clin Oncol 2009, 14, 281-8. Doi:10.1007/ s10147-009-0915-2

12. Platta CS, Khuntia D, Mehta MP, Suh JH. Current treatment strategies for brain metastasis and complications from therapeutic techniques. A review of current literature. Am J Clin Oncol 2010; 33: 398-407. Doi:10.1097/COC.0b013e318194f744

13. Brown PD, Cerhan JH. Same, better, or worse? Neurocognitive effects of radiotherapy for low-grade gliomas remain unknown. Lancet Neurol 2009, 8, 779-81. Doi:10.1016/S1474-4422(09)70205-4

14. Chang EL, Wefel JS, Hess KR, Allen PK, Lang FF, Kornguth DG., et al. Neurocognition in patients with brain metastases treated with radiosurgery or radiosurgery plus whole-brain irradiation: a randomised controlled trial. Lancet Oncol 2009; 10: 1037 44. Doi:10.1016/S1470-2045(09)70263-3

15. Hilverda H, Bosma I, Heimans JJ, Postma TJ, Vandertop WP, Slotman BJ, et al. Cognitive functioning in glioblastoma patients during radiotherapy and temozolomide treatment: Initial findings. J Neurooncol 2010, 97: 8994. Doi:10.1007/s11060-009-9993-2

16. Duffner PK. Risk factors for cognitive decline in children treated for brain tumors. Eur J of Paediatric Neurol. 2010, 106-15. Doi:10.1016/j.ejpn.2009.10.005. 
